\title{
$L$-subshell Coster-Kronig yields of palladium determined via synchrotron-radiation-based high-resolution x-ray spectroscopy
}

\author{
W. Cao, ${ }^{*}$ J. Hoszowska, J.-Cl. Dousse, and Y. Kayser \\ Department of Physics, University of Fribourg, Ch. du Musée 3, CH-1700 Fribourg, Switzerland \\ M. Kavčič, M. Žitnik, K. Bučar, and A. Mihelič \\ J. Stefan Institute, P.O. Box 3000, SI-1001 Ljubljana, Slovenia \\ J. Szlachetko \\ European Synchrotron Radiation Facility (ESRF), 38043 Grenoble, France \\ K. Słabkowska \\ Faculty of Chemistry, Nicholas Copernicus University, 87-100 Toruń, Poland
}

\begin{abstract}
We report on the experimental determination of the palladium $L$-subshell Coster-Kronig (CK) transition yields via high-resolution measurements of the $L \alpha_{1,2}\left(L_{3}-M_{4,5}\right)$ and $L \beta_{1}\left(L_{2}-M_{4}\right)$ x-ray emission lines. The $L$ $\mathrm{x}$-ray spectra were recorded by means of curved crystal spectrometers employing energy-tunable synchrotron radiation for fluorescence production. The $\mathrm{CK}$ yields were derived from the relative $L \mathrm{x}$-ray intensity jumps at the $L$ edges by fitting the fluorescence intensities as a function of the photon energy to the photoionization cross sections. The $L \mathrm{x}$-ray intensities were corrected for solid-state effects which were estimated from the comparison of the measured and theoretical Pd $L$-edge x-ray-absorption spectra. Thanks to high resolution, the partial CK yield $f_{13}^{L_{1} L_{3} M}$ could be extracted from the intensities of the resolved $L \alpha M$ satellite transitions. For $f_{23}$, $f_{12}$, and $f_{13} \mathrm{CK}$ rates, values of $0.164 \pm 0.033,0.047 \pm 0.001$, and $0.730 \pm 0.039$ were found, respectively. For the partial CK yields $f_{13}^{L_{1} L_{3} M}$ and $f_{13}^{L_{1} L_{3} N}$, results of $0.406 \pm 0.023$ and $0.324 \pm 0.032$, respectively, were obtained.
\end{abstract}

\section{INTRODUCTION}

The Coster-Kronig (CK) transitions are special Auger transitions in which the initial and final atomic states are characterized by the presence of a vacancy in the same major shell. In the CK decay, an initial vacancy in the $i$ subshell is transferred to a higher subshell $j$ and a bound electron is ejected simultaneously. The vacancy transfer probability is described by the so-called CK yield $f_{i j}$. The CK rates depend strongly on the overlap between the initial- and final-state wave functions and are very sensitive to electron binding energies as well as solid-state effects [1,2].

Experimental determination of $L$-shell CK yields is challenging. Data are scarce or nonexistent and often suffer from large uncertainties. Most measurements were performed by means of the widely used $K \alpha-L$ x-ray coincidence method $[1,3]$ which has proved to be a powerful tool for determining the $f_{23} \mathrm{CK}$ yield and the $L_{2^{-}}$and $L_{3}$-subshell fluorescence yields $[3,4]$. For the $L_{1}$ subshell, this technique cannot be used since the $K L_{1}$ radiative transition is dipole forbidden. In addition, the $K \alpha$ and $L$ x-ray coincidence detections are difficult to apply to low- $Z$ and mid- $Z$ elements. The alternative photoionization experimental method was limited to the use of radionuclides [5]. About 2 decades ago, with the advent of $\mathrm{x}$-ray synchrotron-radiation sources, the photoionization method based on the selective photoionization of the $L$ subshells by monochromatic synchrotron radiation was intro-

\footnotetext{
*wei.cao@unifr.ch
}

duced [6]. By this mean, the Coster-Kronig vacancy shifts to higher subshells can be switched on and off. By tuning the photon energy across the absorption $L$ edges and recording the X-ray fluorescence lines $[7,8]$ or detecting Auger electrons $[9,10]$, all CK yields can be obtained. So far, measurements of the x-ray fluorescence lines were performed by means of energy-dispersive semiconductor detectors. To the best of our knowledge, high-resolution x-ray emission spectroscopy has not been employed.

The $L$-subshell $C K$ transitions around $Z=48$ have drawn plenty of attention due to the predicted cutoff of the $L_{1}$ $-L_{3} M_{4,5} \mathrm{CK}$ transitions in this region of the periodic table. For elements above the $Z$ value corresponding to the cutoff, the CK transition probabilities vanish because the transitions are energetically forbidden. From theoretical calculations [11], an abrupt cutoff between $Z=49$ and $Z=50$ is expected, however, experimental data suggest that the cutoff is not sharp but extends over a certain range $48 \leq Z \leq 50[12,13]$. Indeed, more experimental data are needed in this mid- $Z$ regime.

In this paper, we report on the high-resolution synchrotron-radiation-based determination of the $L$-subshell $f_{12}, f_{13}$, and $f_{23}$ Coster-Kronig yields of $\operatorname{Pd}(Z=46)$. The palladium $L$-edge absorption spectrum $[14,15]$ and the $L$-shell emission lines [16] are well understood, making it a good candidate for $\mathrm{CK}$ rate determination. The measurements of the $L \alpha_{1,2}\left(L_{3}-M_{4,5}\right)$ and $L \beta_{1}\left(L_{2}-M_{4}\right)$ lines were carried out by means of high-resolution $\mathrm{x}$-ray emission spectroscopy. From the measured x-ray-absorption spectrum, the individual $L$-subshell photoionization cross sections were de- 

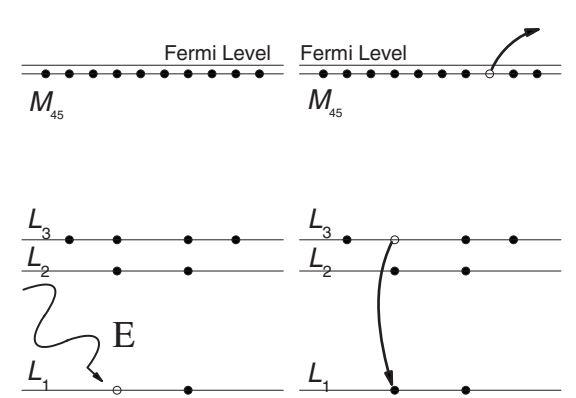

a)

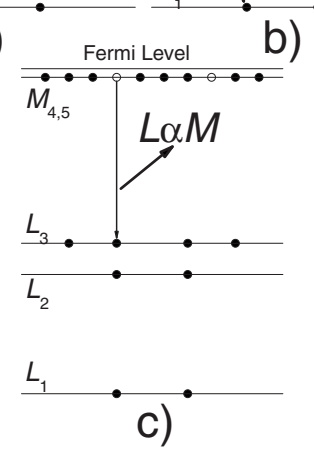

FIG. 1. The $L_{1}-L_{3} M_{4,5}$ Coster-Kronig transition and the $L \alpha M$ satellite x-ray emission. (a) Photoionization of the $L_{1}$ electron. (b) Coster-Kronig process. (c) $L \alpha M$ satellite transition.

termined and compared to theoretical values. The CK yields were derived from the variation of the $L \alpha_{1,2}$ or $L \beta_{1}$ fluorescence line intensities at the absorption $L$ edges due to the onsets of CK vacancy transfers.

Moreover, from the intensities of the resolved $L \alpha M$ satellite $\mathrm{x}$-ray transitions, the partial CK yield $f_{13}^{L_{1} L_{3} M}$ was determined. This was possible because for elements with $Z \leq 91$, the $L_{2}-L_{3} M$ CK transition is energetically forbidden. For illustration, the $L_{1}-L_{3} M_{4,5} \mathrm{CK}$ transition together with the fluorescence decay following the CK process is shown in Fig. 1. This experimental result is new, since the lowresolution $x$-ray spectroscopy method $[8,17]$ cannot give direct information on the $L \alpha M$ satellite lines resulting from the $L_{1}-L_{3} M_{4,5} \mathrm{CK}$ process.

\section{EXPERIMENT}

The experiments were carried out at the bending magnet $\mathrm{x}$-ray absorption fine structure (XAFS) beamline at the Elettra synchrotron, Trieste, Italy. The primary x-ray beam was monochromatized by means of a double-crystal $\mathrm{Si}(111)$ monochromator. To suppress higher photon energies, a Ptcoated mirror was used and the second monochromator crystal was detuned with respect to the first one. The energy bandwidth of the monochromatized radiation was $0.4 \mathrm{eV}$ and the photon flux was $\sim 10^{8} \mathrm{ph} / \mathrm{s}$. The top view of the experimental setup is shown in Fig. 2. For the x-ray-absorption experiment, the dedicated vacuum chamber installed between the second and the third ionization chambers was employed. The $L$-edge x-ray-absorption spectrum was recorded at room temperature in the transmission mode. A selfsupported 1.66- $\mu \mathrm{m}$-thick Pd metallic foil was used. The photon energy was tuned with a step width of $0.5 \mathrm{eV}$ in the range from 3120 to $3700 \mathrm{eV}$. The incident and the transmitted in-

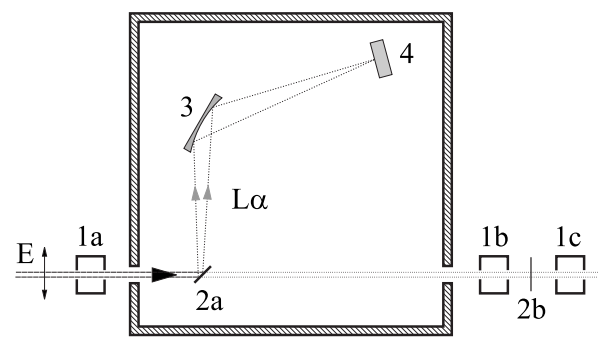

FIG. 2. A top-view scheme of the experimental setup showing the ionization chambers (1), the x-ray spectrometer with the palladium target (2a), silicon crystal (3), and the CCD detector (4). For the $L$-edge absorption measurement, a thin $\mathrm{Pd}$ foil (2b) was mounted perpendicular to the beam in the vacuum chamber installed between the second (1b) and the third (1c) ionization detectors. The first ionization chamber (1a) served to monitor the photon flux during the $\mathrm{x}$-ray emission spectra measurements.

tensities were corrected for the attenuation in the Kapton ${ }^{\mathrm{TM}}$ windows in the beam path as well as for the gas absorption in the ionization chambers. The experimental absorption coefficients were normalized in the $L_{3}$ pre-edge region to the attenuation data from the NIST database [18].

For the high-resolution x-ray emission spectra measurements, the Johansson-type curved crystal spectrometer of Ljubljana [19] was installed in the beamline hutch between the first and the second ionization chambers (see Fig. 2). A $114-\mu \mathrm{m}$-thick metallic palladium sample was mounted in the evacuated spectrometer chamber at $45^{\circ}$ to the incoming beam. The $L \alpha$ x-ray fluorescence lines of Pd were measured using a cylindrically curved $\mathrm{Si}(111)$ crystal in the first order of reflection. The x-ray fluorescence was observed at the angle of $90^{\circ}$ with respect to the horizontally polarized incident photon beam to minimize the background due to elastic scattering. The diffracted $\mathrm{x}$ rays were recorded with a thermoelectrically cooled $\left(-40^{\circ} \mathrm{C}\right)$ back-illuminated chargecoupled device (CCD) camera consisting of 770 $\times 1153$ pixels with a pixel size of $22.5 \times 22.5 \mu \mathrm{m}^{2}$. Positions of the target, crystal, and detector were kept fixed during the whole experiment to ensure constant instrumental parameters. The instrumental energy resolution of the x-ray spectrometer was $\sim 0.3 \mathrm{eV}$. For normalization purposes, the incident photon flux was recorded with the first ionization chamber each $10 \mathrm{~s}$. Beam intensities were corrected for absorption in the gas and the $25-\mu \mathrm{m}$-thick exit Kapton ${ }^{\mathrm{TM}}$ window of the ionization detector. Depending on the incident photon energy and the total photon number, the time to collect an $L \alpha$ x-ray spectrum varied from 50 to $170 \mathrm{~min}$. The fluorescence spectra were calibrated using the reference energy of the $L \alpha_{1}$ x-ray line of 2838.64(5) eV reported in Ref. [20].

A series of $35 L \alpha$ x-ray emission spectra was recorded. The incident-beam energy was tuned from the $L_{3}$ edge (3174 $\mathrm{eV})$ to $3675 \mathrm{eV}$, i.e., $68 \mathrm{eV}$ above the $L_{1}$ edge $(3607 \mathrm{eV})$. Because of software acquisition problems at the end of the experiment, the measurements of the $L \beta_{1} \mathrm{x}$-ray emission lines could not be completed successfully. The latter were carried out later at the ID21 beamline at the ESRF, Grenoble, employing the Fribourg von Hamos curved crystal spectrometer [21]. The same thick Pd target was used and mounted at 


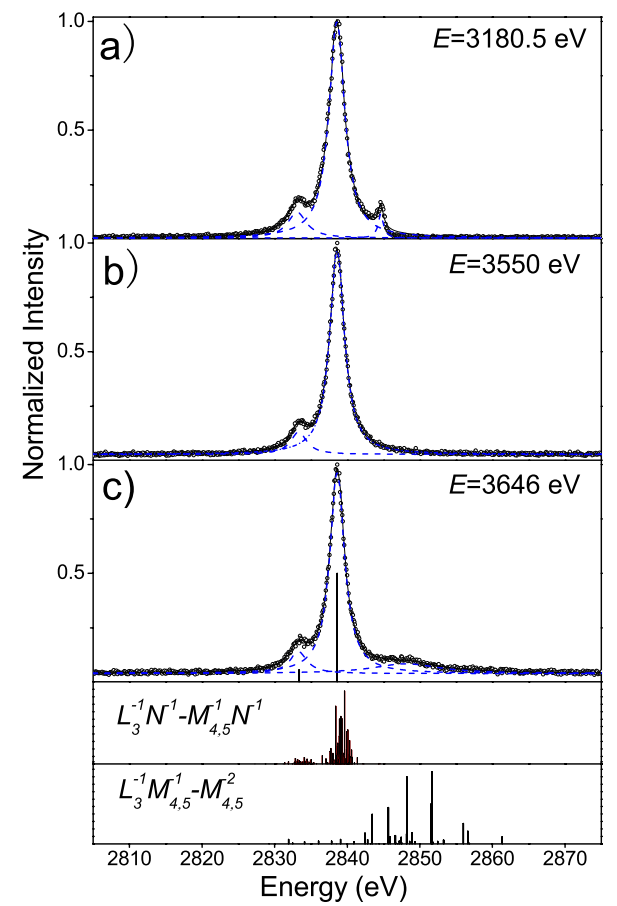

FIG. 3. (Color online) Fitted high-resolution $L \alpha$ X-ray spectra of Pd at different incident-beam energies. Solid thick lines represent the total fit to the experimental data (dots) and dashed lines the individual components. (a) The peak on the high energy side of the $L \alpha$ line is due to RIXS (see text). (b) Shown is the spectrum consisting of the $L \alpha_{1,2}$ diagram lines and the unresolved $N$ satellites. (c) The $L \alpha$ diagram lines, the $N$-satellite, and resolved $M$-satellite lines. The stick spectra in the lower panels correspond to MCDF calculations for the $L \alpha N$ and $L \alpha M$ satellite transitions, respectively.

$45.8^{\circ}$ with respect to the incident beam and a quartz $(1 \overline{1} 0)$ crystal in the first order of reflection was employed. Four $L \beta_{1} \mathrm{x}$-ray spectra were recorded: two at incident-beam energies below and two above the $L_{1}$ edge.

\section{DATA ANALYSIS}

\section{A. Spectra fitting}

The Pd $L \alpha$ X-ray spectra measured at three different photon energies are shown in Fig. 3. The advantage of the highresolution emission spectroscopy is that the individual $L \alpha_{1,2}$ $\mathrm{X}$-ray transitions can be resolved. As a consequence, an elaborated fitting procedure [8] can be avoided. However, the diagram transitions may be accompanied by satellite lines resulting from additional vacancies present in the $N$ and $M$ shells during the x-ray transition. In inner-shell photoionization, these multiple-vacancy configurations may be induced by shake [22] and Coster-Kronig processes. Due to the reduced screening of the nuclear charge, the x-ray satellite lines are shifted in energy with respect to the diagram transitions.

To identify the underlying satellite structure for the fitting procedure, the observed X-ray spectra were compared to multiconfiguration Dirac-Fock (MCDF) calculations [23]. For illustration, $\mathrm{X}$-ray spectra measured at incident-beam energies between the $L_{3}$ and $L_{2}$ edges $(E=3180.5 \mathrm{eV})$, between the $L_{2}$ and $L_{1}$ edges $(E=3550 \mathrm{eV})$, and well above the $L_{1}$ edge $(E=3646 \mathrm{eV})$, together with the results of the MCDF calculations, are depicted in Figs. 3(a)-3(c), respectively. The energies and relative intensities of the individual MCDF multiplet components indicate that the first-order $L \alpha N$ satellite is not resolved from the $L \alpha_{1,2}$ lines. This overlap of the $L \alpha N$ satellite transitions with the diagram lines contributes to a nonlifetime broadening of the latter. In contrast, the $L \alpha M$ satellite is well separated from the parent diagram lines. The calculated average energy shift of the $L \alpha M$ satellite transitions relative to the $L \alpha_{1}$ line amounts to $10.16 \mathrm{eV}$.

From Fig. 3, it can be seen that the $L \alpha$ x-ray emission spectrum depends on the photon energy. For incident-beam energies in the vicinity of the $L_{3}$ edge, the measured $L \alpha$ lines were found to be resonantly enhanced and narrowed. For photon energies slightly above the $L_{3}$ edge, the resonant inelastic $\mathrm{x}$-ray scattering (RIXS) process leads to additional peaks in the $L \alpha_{1,2} \mathrm{X}$-ray spectrum. For illustration, the $L \alpha_{1,2}$ $\mathrm{X}$-ray spectrum observed at an incident-beam energy $6.5 \mathrm{eV}$ above the $L_{3}$ edge is shown in Fig. 3(a). Detailed works on the $L$-edge RIXS for other elements can be found elsewhere $[24,25]$. With increasing photon energy, the $L \alpha N$ satellite lines due to the shake process start to broaden the diagram lines. This nonlifetime broadening becomes more important when additional holes in the $N$ shell are created via the $L_{1,2}-L_{3} N$ CK transitions. For energies above the $L_{1}$ edge, the opening of the $L_{1}-L_{3} M_{4,5} \mathrm{CK}$ channel results in a prominent $M$-shell satellite line [see Fig. 3(c)].

Taking into account the above considerations, the fitting procedure was based on the following assumptions. The $L \alpha_{1,2}$ and $L \beta_{1}$ lines consist of the diagram transitions and the $N$-shell satellites that contribute to the spectral broadening of the Lorentzian profiles, while the $L \alpha M$ satellite transitions are well resolved from the diagram lines. The high-resolution $\mathrm{x}$-ray spectra were fitted by means of the least-square minimization program Peakfit ${ }^{\mathrm{TM}}$. The nonlifetime broadening of the $L \alpha_{1,2}$ and $L \beta_{1}$ lines was accounted for by letting the Lorentzian widths free in the fit and the $L \alpha M$ satellite line was fitted with a single Lorentzian function. An average energy shift of 9.68(16) eV was found for the $L \alpha M$ satellite, comparable to the value of $10.16 \mathrm{eV}$ predicted by MCDF calculations.

\section{B. Self-absorption corrections}

The fitted $L \alpha$ intensities were normalized to the incidentbeam flux and data-collecting time. In addition, to account for the self-absorption of the fluorescence $\mathrm{x}$ rays and attenuation of the incident photon beam in the target, all fitted intensities were multiplied by the following dimensionless correction factor:

$$
F_{\text {corr }}=\frac{\left[\mu(E) / \cos \left(\theta_{1}\right)+\mu_{e} / \cos \left(\theta_{2}\right)\right] t}{1-e^{-\left[\mu(E) / \cos \left(\theta_{1}\right)+\mu_{e} / \cos \left(\theta_{2}\right)\right] t}},
$$

where $\mu(E)$ stands for the total experimental absorption coefficient at the incident-beam energy $E$ and $\mu_{e}$ corresponds to the absorption coefficient at the $L \alpha$ (or $L \beta$ ) energy from [18], $t$ is the target thickness, and $\theta_{1}$ and $\theta_{2}$ are the angles of the 


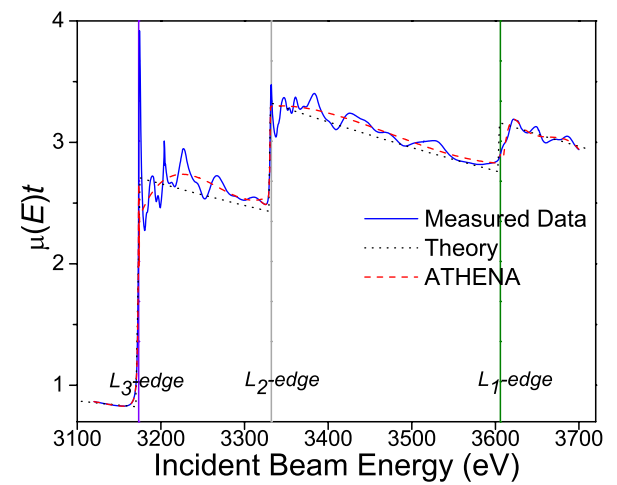

FIG. 4. (Color online) Experimental and theoretical [18] photoabsorptions for a $1.66-\mu \mathrm{m}$-thick palladium sample. For comparison, the smoothed background calculated by means of the ATHENA program is also plotted.

incident and fluorescence photon beams with respect to the normal to the sample surface. Note that for a thick target as the one used in the present experiments, the denominator of Eq. (1) is approximately 1.

\section{Correction of the $L \mathrm{x}$-ray fluorescence intensities for solid-state effects}

The measured $L$ x-ray-absorption spectrum is depicted in Fig. 4, where the total photoabsorption coefficient $\mu$ multiplied by the thickness $t$ of the Pd foil employed for this measurement is plotted as a function of the energy of the incoming photon beam.

The more complex electronic structure of solids compared to atoms results in a different behavior of the absorption coefficient as a function of the photon energy. For Pd, due to the rich hybridization states, the $L$-edge absorption spectrum exhibits x-ray-absorption near-edge structures (XANESs), which can extend as much as $64 \mathrm{eV}$ above the $L_{3}$ edge [14], and for photon energies beyond this region, the extended $\mathrm{X}$-ray-absorption fine structures (EXAFSs) [26]. As a consequence, photoabsorption cross sections at the $L$ edges are either enhanced or diminished compared to the atomlike cross sections for an isolated atom [14,27]. To eliminate the solid-state effects and to extract the real atomic cross section from the measured data, the ATHENA [28,29] program was employed. The calculations were based on the assumption that the EXAFS oscillations are small and that XANES dominates near threshold. The partial cross sections were normalized in the EXAFS regions defined according to [14] and the ATHENA spline algorithm was used. The binding energies $E_{i}$ of the $L_{i}$ subshells were determined from the firstorder derivatives of the rising absorption edges. The calculated "smoothed background" going through the oscillations corresponds to the atomlike photoabsorption cross section $\mu^{a t}(E)$. The latter is compared to the measured photoabsorption cross section $\mu(E)$ in Fig. 4. Since in the energy domain of interest, the coherent- and incoherent-scattering photoionization cross sections are negligibly small compared to the photoelectric cross section, the total photoionization cross sections $\sigma(E)$ and $\sigma^{a t}(E)$ were approximated by the $\mu(E)$ and $\mu^{a t}(E)$, respectively. Furthermore, subtracting from $\sigma(E)$ and $\sigma^{a t}(E)$, the polynomial functions determined by means of the program ATHENA from the data in the three pre-edge regions, the subshell photoionization cross sections $\sigma_{i}(E)$ and $\sigma_{i}^{a t}(E)$ can be obtained.

To account for the solid-state effects, the atomlike $L \alpha$ fluorescence intensity $I_{i}^{a t}(E)$ originating from the decay of a primary vacancy in the $L_{i}$ subshell was derived from the partial experimental intensity $I_{i}(E)$ using the following expression:

$$
I_{i}^{a t}(E)=\frac{I_{i}(E)}{U_{i}(E)},
$$

where the correction function $U_{i}(E)$, as defined in [29], is

$$
U_{i}(E)=1+\frac{\sigma_{i}(E)-\sigma_{i}^{a t}(E)}{\sigma_{i}^{a t}\left(E_{i}\right)} .
$$

It should be noted that $I_{3}(E)$ was deduced in a straightforward way from the fits of the $L \alpha$ line measured with beam energies below the $L_{2}$ edge, whereas for $i=2$ and $i=1$, the $L \alpha$ fluorescence originating from photoionization of the lowerlying subshell(s) was subtracted beforehand (see Sec. IV). A similar but simpler (because of a single absorption edge) procedure was employed to correct the $L \beta_{1}$ intensities.

\section{Parametrization of the $L$-subshell cross sections}

The experimental $L \alpha$ fluorescence intensity $I(E)$ is proportional to the total $L \alpha$ x-ray production cross section $\sigma_{L \alpha}^{X}(E)$ which can be written as

$$
\begin{aligned}
\sigma_{L \alpha}^{X}(E)= & \omega_{L \alpha}\left[\sigma_{3}(E)+f_{23} \sigma_{2}(E)+\left(f_{13}^{L_{1} L_{3} N}+f_{12} f_{23}\right.\right. \\
& \left.\left.+f_{13}^{\prime}\right) \sigma_{1}(E)\right]+\omega_{L \alpha M} f_{13}^{L_{1} L_{3} M} \sigma_{1}(E),
\end{aligned}
$$

where $\sigma_{i}(E)$ are the $L_{i}(i=1,2$, or 3$)$ subshell photoionization cross sections and $f_{13}^{\prime}$ denotes the $L_{1}$ to $L_{3}$ hole-transfer rate resulting from the $L_{1}-L_{3}$ radiative transition. The $\omega_{L \alpha}$ and $\omega_{L \alpha M}$ stand for the $L \alpha$ diagram line and $L \alpha M$ satellite fluorescence yields, respectively. Similarly, the $L \beta$ x-ray production cross section $\sigma_{L \beta}^{X}$ reads

$$
\sigma_{L \beta}^{X}(E)=\omega_{L \beta}\left[\sigma_{2}(E)+f_{12} \sigma_{1}(E)\right],
$$

where $\omega_{L \beta}$ is the partial $L \beta_{1}$ fluorescence yield. The CK rates can be derived from the intensity jumps of the measured $L$ x-ray fluorescence at the $L$ edges, provided that the dependence of the subshell photoionization cross sections $\sigma_{i}(E)$ on the incident photon energy is known.

It is generally assumed that the cross sections vary smoothly with photon energy and within a certain energy range the dependence can be described by a power law $[8,30]$. In the mid- $Z$ range, the following analytical function can be used:

$$
\sigma_{i}^{a t}(E)=a_{i}[E(\mathrm{keV})]^{b_{i}}
$$

where $a_{i}$ and $b_{i}$ are the fitting parameters for a particular subshell $i$. The dependence is different for individual $L_{i}$ subshells and changes in the vicinity of the $L$ edges.

The XANES and EXAFS modulations in our experimental absorption spectrum did not permit a reliable power-law 
TABLE I. Analytical fit to the $L$-subshell photoabsorption cross sections of palladium (in units of $10^{3} \mathrm{~cm}^{2} \mathrm{~g}^{-1}$ ).

\begin{tabular}{lcc}
\hline \hline Ionized subshell & Fitted function & Fitted function $^{\mathrm{a}}$ \\
\hline$L_{3}$ & $16.483[E(\mathrm{keV})]^{-2.456}$ & $11.413[E(\mathrm{keV})]^{-2.158}$ \\
$L_{2}$ & $8.639[E(\mathrm{keV})]^{-2.442}$ & $11.710[E(\mathrm{keV})]^{-2.711}$ \\
$L_{1}$ & $1.082[E(\mathrm{keV})]^{-1.253}$ & $8.739[E(\mathrm{keV})]^{-2.937}$ \\
\hline \hline
\end{tabular}

${ }^{\mathrm{a}}$ Fit to the data from [18] (see text).

fit to the data. In order to extract the $a_{i}$ and $b_{i}$ parameters, we have resorted to the theoretical absorption coefficients $[18,31]$. The values from the NIST database [18] were found to be in good agreement with our experimental data. However, it should be noted that the photoabsorption cross sections in the energy region of interest are calculated with an energy step of $\sim 100 \mathrm{eV}$ and a smaller energy grid is obtained from an interpolation algorithm. This fact affects the accuracy of the fit. An alternative is to use the data given by Ref. [31] in which a smaller energy grid is applied. Both sets of calculated data agree quite well below the $L_{1}$ edge. For the $L_{1}$ absorption coefficient, however, values from [31] were found to be $\sim 22 \%$ smaller than those from [18] and the present experimental results. Therefore, to obtain the $a_{i}$ and $b_{i}$ parameters, the following approach was adopted. First, the parameters $a_{i}$ and $b_{i}$ for the $L_{3}$ and $L_{2}$ absorption coefficients and the $b_{1}$ were extracted from the data of Henke et al. [31] using Eq. (5). To get $a_{1}$, data from [18] were fitted with a fixed value of the $b_{1}$ parameter obtained from the former fit. The obtained fitting parameters are collected in Table I. It can be seen that $b_{2}$ and $b_{3}$ are almost the same, while $a_{2}$ is about half of $a_{3}$. These values are consistent with those predicted by Jitschin and Stötzel [17] for elements with $47 \leq Z$ $\leq 51$. For comparison, the fitted parameters for the data from Ref. [18], calculated with an interpolated energy step of $2 \mathrm{eV}$, are also listed in Table I.

The experimental and theoretical [18] photoabsorption coefficients are shown on the same plot of Fig. 4. Due to the $L_{3}$ - and $L_{2}$-edge "white lines" which originate from photoexcitation to the unfilled valence states as well as the oscillatory structures resulting from solid-state effects, differences between the theoretical and experimental absorption data can be observed. However, the calculated "smoothed values," for which the changes in the absorption cross sections due to XANES and EXAFS were averaged out, are found in reasonable agreement with theory. The minor deviations from the power-law dependence of the cross sections based on the independent-particle approximation are related to electroncorrelations effects [30].

\section{RESULTS AND DISCUSSION}

The $f_{23} \mathrm{CK}$ yield was extracted from the $L \alpha$ intensity jump at the $L_{2}$ edge. First, the atomlike fluorescence intensity below the $L_{2}$ edge was fitted with the following expression:

$$
I_{3}^{a t}(E)=C a_{3} \omega_{L \alpha}[E(\mathrm{keV})]^{b_{3}},
$$

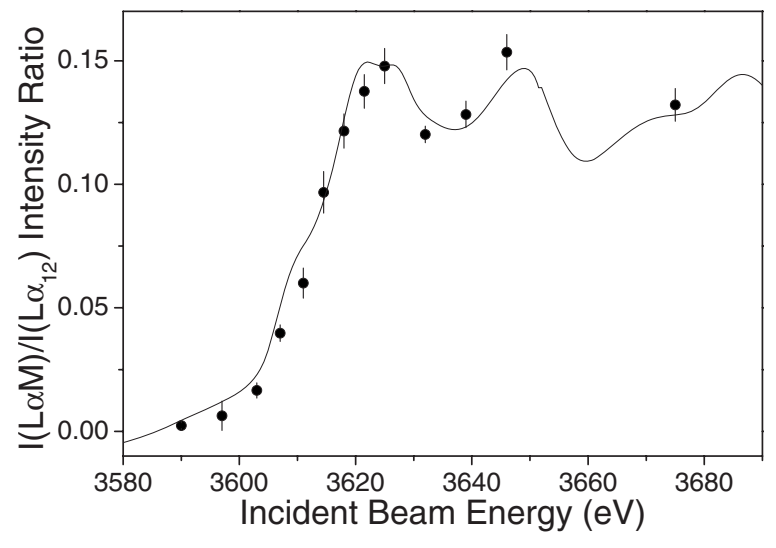

FIG. 5. Ratio of the $L \alpha M$ satellite line to the diagram line vs the photon energy. Solid line represents the experimental photoionization cross section $\sigma_{1}(E)$ normalized to the ratio at an energy of $E$ $=3625 \mathrm{eV}$.

yielding the parameter $A_{3}=C a_{3} \omega_{L \alpha}$, where $C$ is the instrumental proportionality constant. To derive the $\mathrm{CK}$ contribution, the fluorescence intensities $I_{3}^{a t}(E)$ for energies above the $L_{2}$ edge were extrapolated and subtracted from the $I(E)$ data. Since the $L_{3}$ EXAFS oscillations are very small for photon energies $E>E_{2}$, we have assumed that the extrapolated values $I_{3}(E)=I_{3}^{a t}(E)$. The obtained $I_{2}(E)$ intensities were converted using the correction function $U_{2}(E)$ to atomlike values $I_{2}^{a t}(E)$. The latter were fitted with a similar function providing the parameter $A_{2}=f_{23} C a_{2} \omega_{L \alpha}$. The value for $f_{23}$ was then derived from the relation

$$
f_{23}=\frac{A_{2} a_{3}}{A_{3} a_{2}} .
$$

For the $f_{13}$ yield, first the partial CK yield $f_{13}^{L_{1} L_{3} N}$ was determined. To this end, a similar approach as for $f_{23}$ was adopted. The extrapolated values for $I_{2}^{a t}(E)$ and $I_{3}^{a t}(E)$ were first subtracted from $I(E)$ giving the intensity $I_{1}^{L_{1} L_{3} N}(E)$, and after correction with $U_{1}(E)$, the corresponding atomlike function $I_{1}^{L_{1} L_{3} N^{a t}}(E)$ was fitted to obtain the value for the parameter $A_{1}^{L_{1} L_{3} N}$,

$$
A_{1}^{L_{1} L_{3} N}=\left(f_{13}^{L_{1} L_{3} N}+f_{12} f_{23}+f_{13}^{\prime}\right) C a_{1} \omega_{L \alpha} .
$$

Subsequently, the partial CK $f_{13}^{L_{1} L_{3} N}$ yield was calculated using the following expression:

$$
f_{13}^{L_{1} L_{3} N}=\frac{A_{1}^{L_{1} L_{3} N} a_{3}}{A_{3} a_{1}}-\left(f_{12} f_{23}+f_{13}^{\prime}\right) .
$$

The partial $f_{13}^{L_{1} L_{3} M} \mathrm{CK}$ yield was determined from the $M$-satellite intensities employing the following formula:

$$
f_{13}^{L_{1} L_{3} M}=\frac{A_{1}^{L_{1} L_{3} M} a_{3}}{A_{3} a_{1}},
$$

where $A_{1}^{L_{1} L_{3} M}=f_{13}^{L_{1} L_{3} M} C a_{1} \omega_{L \alpha M}$ is the fitting parameter for the $L \alpha M$ satellite fluorescence intensity $I_{1}^{L_{1} L_{3} M^{a t}}(E)$. Only data 
for incident photon energies above $3618 \mathrm{eV}$ were considered. The CK channel is fully open then and the $M$ satellite to diagram line intensity ratios normalized to the experimental photoionization cross section $\sigma_{1}(E)$ are constant (see Fig. 5). For beam energies in the vicinity of the $L_{1}$ edge, a smooth rise of the ratio with photon energy can be observed. This variation is mainly related to the finite lifetime of the $L_{1}$ vacancy state.

A similar method was applied to deduce the $f_{12}$ yield using the following formula:

$$
f_{12}=\frac{A_{1}^{\prime} a_{2}}{A_{2}^{\prime} a_{1}},
$$

where $A_{1}^{\prime}=f_{12} C^{\prime} a_{1} \omega_{L \beta}$ and $A_{2}^{\prime}=C^{\prime} a_{2} \omega_{L \beta}$.

Further, to account for the differences in the fluorescence yields of the $L_{3}^{-1} \rightarrow M_{4,5}^{-1}$ diagram line and the $L_{3}^{-1} M_{4,5}^{-1}$ $\rightarrow M_{4,5}^{-2}$ X-ray transition in the presence of an additional hole in the $M_{4,5}$ shell, the ratio $\omega_{L \alpha M} / \omega_{L \alpha}$ was estimated. The following relations based on a statistical weighting procedure similar to the one described in [32] were employed:

$$
\begin{gathered}
\omega_{L \alpha}=\frac{\Gamma_{L \alpha}}{\Gamma_{3}}, \\
\omega_{L \alpha M}=\frac{\frac{9}{10} \Gamma_{L \alpha}}{\Gamma_{L \beta}+\Gamma_{L \iota}+\Gamma_{L_{3} M_{123} M_{123}}^{A}+\Gamma_{L_{3} M_{123^{N}}}^{A}+\frac{9}{10}\left(\Gamma_{L \alpha}+\frac{8}{9} \Gamma_{L_{3} M_{45} M_{45}}^{A}+\Gamma_{L_{3} M_{123} M_{45}}^{A}+\Gamma_{L_{3} M_{45} N}^{A}\right)+\Gamma_{L_{3} N N}^{A}},
\end{gathered}
$$

where $\Gamma_{3}$ is the $L_{3}$-subshell total transition rate, $\Gamma_{L \alpha}$ the total $L \alpha$ x-ray emission rate, $\Gamma_{L \beta}$ the sum of the x-ray emission rates of the $L \beta_{2}, L \beta_{6}$, and $L \beta_{15}$ transitions, and $\Gamma^{A}$ stands for the radiationless transition rates. In the calculation, the radiative transition rates $\Gamma_{L \alpha}, \Gamma_{L \beta}$, and $\Gamma_{L \iota}$ were taken from the theoretical calculations of Scofield [33] and the Auger probabilities were interpolated from the values quoted in Ref. [34] for $\mathrm{Rh}(Z=45)$ and $\mathrm{Ag}(Z=47)$. A $\omega_{L \alpha M} / \omega_{L \alpha}$ ratio of 1.014 was found. This value denotes a negligibly small difference between the $L \alpha$ and $L \alpha M$ satellite line fluorescence yields.

As already mentioned, $N$ - and $M$-shell satellites are due not only to $\mathrm{CK}$ transitions, but may also originate from the shake process. In the shake process, the primary electron is ejected rapidly and due to the sudden change of the central potential a subsequent excitation of a second electron to a bound state or to the continuum takes place. To delineate the role played by shake contributions to $L$ x-ray fluorescence intensity changes, the shake probabilities for $\mathrm{Pd}$ were calculated in the framework of the sudden approximation model [35] using self-consistent Dirac-Fock wave functions from the code of [36]. More information on the calculations can be found in [37]. The calculated $N$-shake probability when a single vacancy is created in the $L_{i}$ subshell was found to be $P_{N}=0.202$, where for $N_{4,5}$ electrons $P_{N_{4,5}}=0.184$. For the $M$-shell, the shake probabilities of $P_{M}=0.0088$ for the initial single vacancy in the $L_{1}$ subshell and $P_{M}=0.0092$ in the $L_{2,3}$ subshells, respectively, were obtained.

Although the shake probabilities of $N_{4,5}$ subshell electrons can reach as much as $18 \%$, this contribution is constant over the whole range of incident photon energies and thus does not influence the $L$ x-ray intensity jumps. Concerning the $M$-shake probability, according to the sudden approximation model, the above calculated values are valid for primary photon energies higher than about 10 times the $L M$ doubleionization threshold, i.e., $\sim 6970 \mathrm{eV}$ [38]. This means that the relative satellite intensity due to $M$-shake events is even less than $1.8 \%$ and thus is negligibly small. Indeed, the observed $L \alpha M$ satellites originate from the $L_{1}-L_{3} M_{4,5} \mathrm{CK}$ transitions.

Figure 6 shows the experimental values and fitted curves of the atomlike fluorescence intensities $I^{a t}(E)$ given by

$$
I^{a t}(E)=I_{3}^{a t}(E)+I_{2}^{a t}(E)+I_{1}^{a t}(E)
$$

where $I_{1}^{a t}(E)=I_{1}^{L_{1} L_{3} N^{a t}}(E)+I_{1}^{L_{1} L_{3} M^{a t}}(E)$ stands for the atomlike fluorescence intensity due to a hole in the $L_{1}$ subshell. The fluorescence intensity jumps at the $L$ edges corresponding to the particular $\mathrm{CK}$ transition yields are also depicted. For the

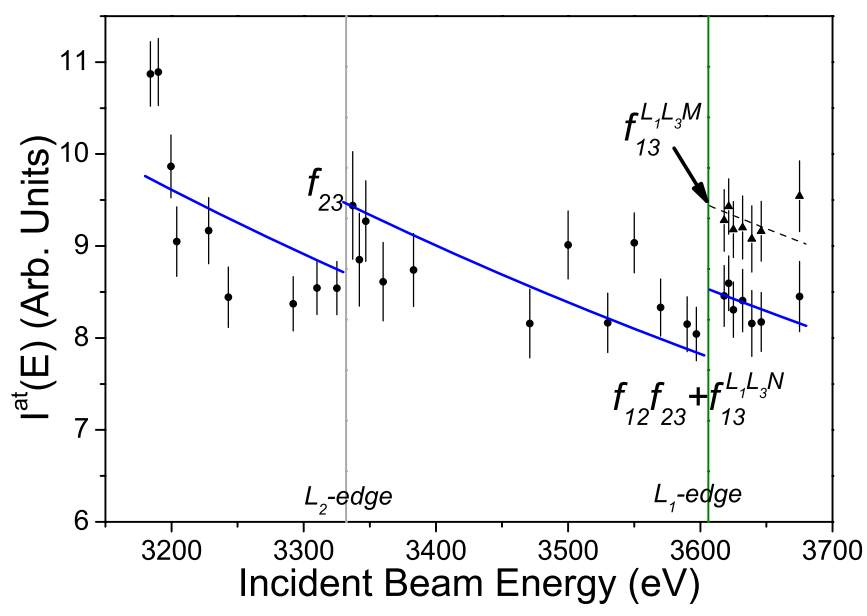

FIG. 6. (Color online) Atomlike fluorescence intensities $I^{a t}(E)$ plotted against the incident-beam energy. Experimental points are depicted with solid circles, while solid lines represent the fitted curves. The data set used to derive the partial Coster-Kronig yield $f_{13}^{L_{1} L_{3} M}$ is plotted separately (solid triangles), while dashed line stands for the fitted values. Vertical lines indicate the $L$ edges. 
TABLE II. Coster-Kronig transition yields of palladium.

\begin{tabular}{|c|c|c|c|c|c|}
\hline & $f_{23}$ & $f_{12}$ & $f_{13}$ & $f_{13}^{L_{1} L_{3} N}$ & $f_{13}^{L_{1} L_{3} M}$ \\
\hline Present & $0.164(33)$ & $0.047(1)$ & $0.730(39)$ & $0.324(32)$ & $0.406(23)$ \\
\hline Present $^{\mathrm{a}}$ & $0.130(30)$ & $0.072(4)$ & $0.743(45)$ & $0.307(34)$ & $0.436(29)$ \\
\hline Theory ${ }^{b}$ & 0.154 & 0.065 & 0.75 & 0.095 & 0.655 \\
\hline Theory ${ }^{c}$ & & 0.103 & 0.58 & 0.163 & 0.418 \\
\hline Ag [8] & $0.18(3)$ & $0.14(3)$ & $0.58(5)$ & & \\
\hline $\mathrm{Ag}[9]$ & $0.16(3)$ & $0.044(4)$ & $0.61(5)$ & & \\
\hline Ag Theory ${ }^{b}$ & 0.155 & 0.068 & 0.74 & 0.108 & 0.634 \\
\hline Ag Theory ${ }^{c}$ & & 0.109 & 0.56 & 0.191 & 0.364 \\
\hline
\end{tabular}

${ }^{\mathrm{a}}$ These results were derived using the fitting parameters listed in the second column of Table I.

${ }^{\mathrm{b}}$ Calculations by Chen et al. $[2,34]$.

${ }^{\mathrm{c}}$ Calculations with adjusted $L_{1}-L_{3} M$ transition rates (see text).

$f_{23}$ CK rate, a value of $0.164 \pm 0.033$ was calculated using Eq. (7). The partial CK yields of $0.324 \pm 0.032$ for $f_{13}^{L_{1} L_{3} N}$ and $0.406 \pm 0.023$ for $f_{13}^{L_{1} L_{3} M}$ were deduced adopting for the $f_{12}$ yield the value of $0.047(1)$ from Eq. (11). This $f_{12}$ rate is close to the value of 0.044(4) reported by Sorensen et al. for $\mathrm{Ag}$ [9] and to the calculated prediction of 0.065 of Chen et al. [2] but smaller than the result of 0.1 reported by Krause [39]. The total CK probability $f_{13}=f_{13}^{L_{1} L_{3} N}+f_{13}^{L_{1} L_{3} M}$ amounts to $0.730 \pm 0.039$. It should be noted that since the competing intrashell radiative transitions are negligibly small compared to the CK process [10], the value of $f_{13}^{\prime}$ was not taken into consideration in the calculations.

The present results together with the theoretical predictions are listed in Table II and the $f_{13} \mathrm{CK}$ probabilities are compiled in Fig. 7. In Table II, the CK yields calculated using the fitting parameters obtained from the interpolated photoabsorption coefficients of Ref. [18] are also presented. It can be seen that both sets of data agree quite well with each other. To the best of our knowledge, no other experimental data for the CK yields of Pd exist. Compared to the experimental values reported for $\mathrm{Ag}[8,10]$, the total $f_{13}$ rate for Pd is found to be $\sim 20 \%$ higher.

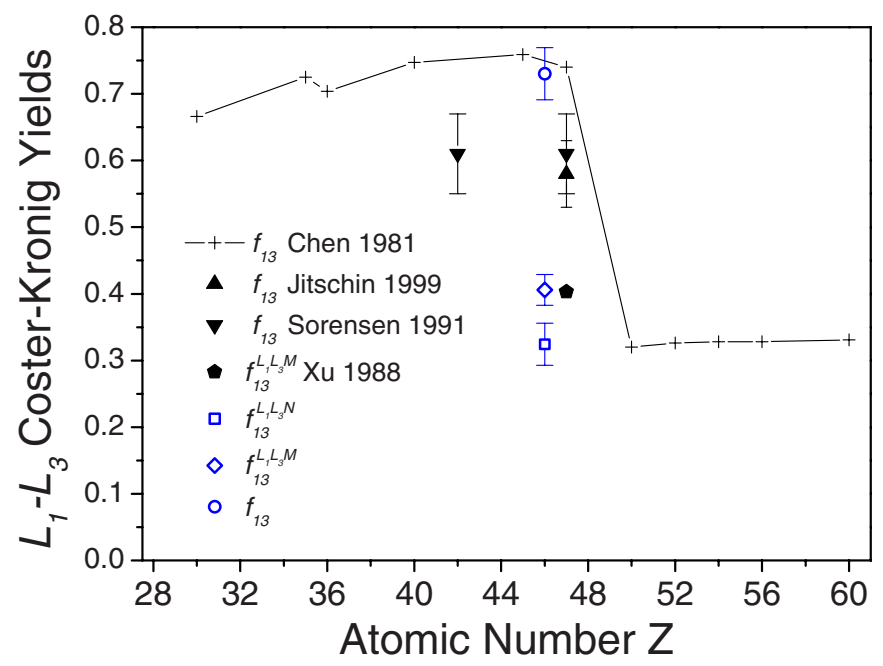

FIG. 7. (Color online) Present $f_{13} \mathrm{CK}$ rates of Pd (open symbols) compared to theoretical values from [2] and available experimental data for neighboring elements, Mo [10] and $\mathrm{Ag}[8,10,40]$.
The $f_{23}$ CK rate is consistent within the experimental uncertainty with the theoretical value from [2]. The present total CK probability $f_{13}$ is also comparable with the calculations of Chen et al. [2], but the relative contributions of the $f_{13}^{L_{1} L_{3} N}$ and $f_{13}^{L_{1} L_{3} M}$ CK yields are quite different. In fact, the partial CK transition $L_{1}-L_{3} N$ for Pd is close to the values of the semiempirical fit of Krause [39] for elements in the range $50 \leq Z \leq 74$ for which the $L_{1}-L_{3} M$ CK transitions are energetically forbidden [34].

The discrepancies between the theoretical and experimental values can be partly ascribed to the overestimated theoretical $L_{1}-L_{3} M$ transition rates. As already pointed out by Chen et al. [41] for the case of Ag, adjusting the $L_{1}$ $-L_{3} M_{4,5}$ rate by a factor 0.4 leads to a better agreement of the experimental and theoretical $L_{1}$ level widths. Indeed, for $\mathrm{Pd}$, the calculated $L_{1}$ width is $6.8 \mathrm{eV}$ [2], while a value of 3.9 $\mathrm{eV}$ is recommended by Campbell and Papp [42]. We have therefore rescaled the theoretical CK transition width $\Gamma_{L_{1} L_{3} M}^{\mathrm{CK}}$ using the following transformation:

$\Gamma_{1}=\Gamma_{t o t}^{A}+\Gamma_{t o t}^{R}=\frac{\Gamma_{t o t}^{A}}{1-\omega_{1}} \rightarrow \Gamma_{1}^{e x p}=\frac{\Sigma_{(-)} \Gamma^{A}+\Gamma_{L_{1} L_{3} N}^{\mathrm{CK}}+\eta \Gamma_{L_{1} L_{3} M}^{\mathrm{CK}}}{1-\omega_{1}}$,

where $\Gamma_{1}$ and $\Gamma_{1}^{e x p}$ represent the theoretical and experimental widths of the atomic level $L_{1}, \Gamma_{t o t}^{A}$ and $\Gamma_{t o t}^{R}$ the sums of the widths of all Auger and radiative transitions decaying $L_{1}$-vacancy states, $\omega_{1}$ the partial fluorescence yield of the $L_{1}$ subshell, $\Sigma_{(-)} \Gamma^{A}$ the total $L_{1}$ Auger width minus the widths of the $L_{1}-L_{3} M$ and $L_{1}-L_{3} N$ CK transitions, and $\eta$ is a constant. The widths of the radiationless transitions were obtained from the Auger transition probabilities reported by Chen et al. [34], whereas the width $\Gamma_{1}$ and fluorescence yield $\omega_{1}$ were taken from Ref. [2] and the experimental width $\Gamma_{1}^{\exp }$ from Ref. [42]. The adjustment factor $\eta$ can be deduced from Eq. (14),

$$
\eta=\frac{\left(1-\omega_{1}\right) \Gamma_{1}^{e x p}-\Sigma_{(-)} \Gamma^{A}-\Gamma_{L_{1} L_{3} N}^{\mathrm{CK}} .}{\Gamma_{L_{1} L_{3} M}^{\mathrm{CK}}} .
$$

As Chen's calculations reported in [2,34] concern only the neighbor elements $\mathrm{Rh}(Z=45)$ and $\mathrm{Ag}(Z=47)$, the factors $\eta$ 
were first computed for these two elements. Values of 0.425 and 0.325 , respectively, were obtained from which the factor $\eta$ corresponding to Pd was then determined by interpolation. Finally, the adjusted CK yields $f_{12}^{a d j}, f_{13}^{L_{1} L_{3} M, a d j}$, and $f_{13}^{L_{1} L_{3} N, \text { adj }}$ given in Table II were calculated by means of the following relations:

$$
\begin{gathered}
f_{12}^{a d j}=\frac{\Gamma_{1}}{\Gamma_{1}^{e x p}} f_{12}, \\
f_{13}^{L_{1} L_{3} M, a d j}=\frac{\eta \Gamma_{1}}{\Gamma_{1}^{\text {exp }}} f_{13}^{L_{1} L_{3} M}, \\
f_{13}^{L_{1} L_{3} N, a d j}=\frac{\Gamma_{1}}{\Gamma_{1}^{\text {exp }} f_{13}^{L_{1} L_{3} N} .}
\end{gathered}
$$

As shown in Table II, the obtained $f_{13}^{L_{1} L_{3} M, \text { adj }}$ value is close to our experimental result, but the $f_{13}^{L_{1} L_{3} N, a d j}$ rate is still a factor of 2 smaller. This results in a twice as large experimental $L_{1}-L_{3} N$ to $L_{1}-L_{3} M$ CK yields ratio compared to the calculated one. A similar trend can be also observed for Ag. Calculations including many-body and solid-state effects for the radiative and nonradiative $L_{1}$-subshell decay rates and more experimental data in this $Z$ region would certainly help to unravel the observed discrepancies.

The estimated uncertainties of the derived Pd CK yields of $10 \%-25 \%$ stem mainly from the uncertainties of the fitting parameters $A_{i}$. With the current method, the solidstate effects could be diminished to a large extent and the effect of the parameters $b_{i}$ was found to be small. However, the differences between the experimental and relativistic Hartree-Slater (RHS) calculations, caused by the electroncorrelation effects [17], can be as much as $3 \%$ below the $L_{2}$ edge and $4 \%$ above, respectively. These differences also affect the uncertainties of the $L \alpha$ intensities extracted from the extrapolation of the fitted photoionization cross sections beyond the individual $L$-subshell edges, in particular the $L_{3}$ edge. It should be also noted that numerical results of different theoretical predictions can vary up to $2 \%$ [43]. All of these effects can lead to uncertainties up to $30 \%$ for the CK yields [3].

\section{CONCLUDING REMARKS}

We have carried out a determination of the Coster-Kronig transition yields for Pd via synchrotron-radiation-based highresolution measurements of the $L \mathrm{x}$-ray emission lines. By means of a fitting procedure, making use of the power-law dependence of the photoionization cross sections on the primary photon energy, the $f_{23}$ and $f_{13}$ CK yields were derived from the relative $L \alpha$ intensity jumps at the $L$ edges and the $f_{12}$ rate from the $L \beta_{1}$ intensity jump at the $L_{1}$ edge. The measured $L$-edge x-ray-absorption spectrum was compared to the theoretical cross sections and served to correct the $L$ $\mathrm{x}$-ray intensities for solid-state effects. Thanks to the resolved $L \alpha M$ satellite lines, the partial $L_{1}-L_{3} M_{4,5}$ CK transition yield could be determined. The differences in the transition probabilities for the $M$-satellite lines and the parent diagram lines were accounted for. The sensitivity of the CK yields to the parametrization of the photoabsorption cross sections was also considered. Our result for the total $f_{13} \mathrm{CK}$ yield is consistent with the theoretical calculations of Chen et al. [2], however, the obtained value is higher compared to the experimental ones for neighboring elements.

The discrepancies between the present and theoretical values for the relative contributions of the partial $L_{1}-L_{3} N$ and $L_{1}-L_{3} M$ CK yields point to the need of including manybody and solid-state effects for the radiative and nonradiative $L_{1}$-subshell decay rates. While the total $f_{13}$ Coster-Kronig yields are available, the experimental data for partial $f_{13} \mathrm{CK}$ transition rates are scarce or not existing. More experimental values obtained from measurements with the synchrotronradiation-based high-resolution x-ray emission or Augerelectron spectroscopy techniques for the relative contributions of the partial $L_{1}-L_{3} N$ and $L_{1}-L_{3} M$ CK yields are called for.

\section{ACKNOWLEDGMENTS}

The authors would like to thank L. Olivi and G. Vlaic of the beamline XAFS at Elettra and the staff of the ESRF beamline ID21 for providing our experiment with high quality beams. The financial support of the Swiss National Science Foundation (Grant No. 200020-116603) and of the Slovenian Ministry of Higher Education, Science, and Technology (Program No. P1-0112) are acknowledged.
[1] W. Bambynek, B. Crasemann, R. W. Fink, H.-U. Freund, H. Mark, C. D. Swift, R. E. Price, and P. V. Rao, Rev. Mod. Phys. 44, 716 (1972).

[2] M. H. Chen, B. Crasemann, and H. Mark, Phys. Rev. A 24, 177 (1981).

[3] J. L. Campbell, At. Data Nucl. Data Tables 85, 291 (2003).

[4] R. W. Dunford, E. P. Kanter, B. Krässig, S. H. Southworth, L. Young, P. H. Mokler, Th. Stöhlker, S. Cheng, A. G. Kochur, and I. D. Petrov, Phys. Rev. A 74, 062502 (2006).

[5] J. L. Campbell, At. Data Nucl. Data Tables 95, 115 (2009), and references therein.
[6] W. Jitschin, G. Materlik, U. Werner, and P. Funke, J. Phys. B 18, 1139 (1985).

[7] U. Werner and W. Jitschin, Phys. Rev. A 38, 4009 (1988).

[8] W. Jitschin, R. Stötzel, T. Papp, and M. Sarkar, Phys. Rev. A 59, 3408 (1999).

[9] S. L. Sorensen, R. Carr, S. J. Schaphorst, S. B. Whitfield, and B. Crasemann, Phys. Rev. A 39, 6241 (1989).

[10] S. L. Sorensen, S. J. Schaphorst, S. B. Whitfield, B. Crasemann, and R. Carr, Phys. Rev. A 44, 350 (1991).

[11] S. Puri, D. Mehta, B. Chand, N. Singh, and P. N. Trehan, X-Ray Spectrom. 22, 358 (1993). 
[12] B. L. Doyle and S. M. Shafroth, Phys. Rev. A 19, 1433 (1979).

[13] E. Rosato, Nucl. Instrum. Methods Phys. Res. B 15, 591 (1986).

[14] T. K. Sham, Phys. Rev. B 31, 1888 (1985).

[15] L. C. Witjens, J. H. Bitter, A. J. van Dillen, K. P. de Jong, and F. M. F. de Groot, Phys. Chem. Chem. Phys. 6, 3903 (2004).

[16] M. Czarnota et al., Braz. J. Phys. 36, 546 (2006).

[17] W. Jitschin and R. Stötzel, Phys. Rev. A 58, 1221 (1998).

[18] http://physics.nist.gov/xcom

[19] M. Kavčič, A. G. Karydas, and Ch. Zarkadas, Nucl. Instrum. Methods Phys. Res. B 222, 601 (2004).

[20] R. D. Deslattes, E. G. Kessler, Jr., P. Indelicato, L. de Billy, E. Lindroth, and J. Anton, Rev. Mod. Phys. 75, 35 (2003).

[21] J. Hoszowska, J.-Cl. Dousse, J. Kern, and C. Rhême, Nucl. Instrum. Methods Phys. Res. A 376, 129 (1996).

[22] T. Åberg, Phys. Rev. 156, 35 (1967).

[23] K. Słabkowska and M. Polasik, Radiat. Phys. Chem. 75, 1471 (2006).

[24] M. A. MacDonald, S. H. Southworth, J. C. Levin, A. Henins, R. D. Deslattes, T. LeBrun, Y. Azuma, P. L. Cowan, and B. A. Karlin, Phys. Rev. A 51, 3598 (1995).

[25] M. Žitnik, M. Kavčič, K. Bučar, A. Mihelič, M. Štuhec, J. Kokalj, and J. Szlachetko, Phys. Rev. A 76, 032506 (2007).

[26] J. J. Rehr and R. C. Albers, Rev. Mod. Phys. 72, 621 (2000).
[27] J. E. Müller and J. W. Wilkins, Phys. Rev. B 29, 4331 (1984).

[28] http://cars9.uchicago.edu/ ravel/software/exafs/

[29] B. Ravel and M. Newville, Phys. Scr., T 115, 1007 (2005).

[30] W. Jitschin, U. Werner, G. Materlik, and G. D. Doolen, Phys. Rev. A 35, 5038 (1987).

[31] B. L. Henke, E. M. Gullikson, and J. C. Davis, At. Data Nucl. Data Tables 54, 181 (1993); online calculations at http:// henke.lbl.gov/optical_constants/

[32] F. P. Larkins, J. Phys. B 4, 1 (1971).

[33] J. H. Scofield, At. Data Nucl. Data Tables 14, 121 (1974).

[34] M. H. Chen, B. Crasemann, and H. Mark, At. Data Nucl. Data Tables 24, 13 (1979).

[35] T. Mukoyama and K. Taniguchi, Phys. Rev. A 36, 693 (1987).

[36] K. G. Dyall, J. Phys. B 16, 3137 (1983).

[37] J.-Cl. Dousse and J. Hoszowska, Phys. Rev. A 56, 4517 (1997).

[38] T. D. Thomas, Phys. Rev. Lett. 52, 417 (1984).

[39] M. O. Krause, J. Phys. Chem. Ref. Data 8, 307 (1979).

[40] J. Q. Xu and E. Rosato, Phys. Rev. A 37, 1946 (1988).

[41] M. H. Chen, B. Crasemann, M. Aoyagi, and H. Mark, Phys. Rev. A 15, 2312 (1977).

[42] J. L. Campbell and T. Papp, At. Data Nucl. Data Tables 77, 1 (2001).

[43] J. H. Hubbell, Phys. Med. Biol. 51, R245 (2006). 